\title{
Searching for space-time variation of the fine structure constant using QSO spectra: overview and future prospects
}

\author{
J. C. Berengut ${ }^{1}$, V. A. Dzuba ${ }^{1}$, V. V. Flambaum ${ }^{1}$, J. A. King ${ }^{1}$, M. G. \\ Kozlov $^{2}$, M. T. Murphy ${ }^{3}$ and J. K. Webb ${ }^{1}$ \\ ${ }^{1}$ School of Physics, University of New South Wales, Sydney, NSW 2052, Australia \\ ${ }^{2}$ Petersburg Nuclear Physics Institute, Gatchina, 188300, Russia \\ ${ }^{3}$ Centre for Astrophysics and Supercomputing, Swinburne University of Technology, \\ Victoria 3122, Australia
}

Current theories that seek to unify gravity with the other fundamental interactions suggest that spatial and temporal variation of fundamental constants is a possibility, or even a necessity, in an expanding Universe. Several studies have tried to probe the values of constants at earlier stages in the evolution of the Universe, using tools such as big-bang nucleosynthesis, the Oklo natural nuclear reactor, quasar absorption spectra, and atomic clocks (see, e.g. Flambaum \& Berengut (2009)).

Comparison of atomic transition frequencies on Earth and in quasar absorption spectra can be used to measure variation of the fine-structure constant $\alpha=e^{2} / \hbar c$ over the last 10 billion years or so. The "many-multiplet" method Dzuba (1999) improves sensitivity to variation in $\alpha$ by more than an order of magnitude compared to the old "alkali-doublet" method. Study of three independent samples of data, containing 143 absorption systems over the redshift range $0.2<z_{\text {abs }}<4.2$, gives a variation of $\Delta \alpha / \alpha=(-0.57 \pm 0.11) \times 10^{-5}$ Murphy et al (2004). Our method and calculations have been used by other groups to analyse different data sets from different telescopes Srianand et al (2004); their results indicate no variation of $\alpha$. However a re-analysis of the same data, which included 23 absorption systems from VLT/UVES, increased the error previously reported by a factor of six (Murphy et al (2007), Murphy et al (2008)).

The studies can be improved by utilising more atomic transitions that are seen in quasar spectra, however in many cases this is hampered by a lack of accurate laboratory frequency measurements. We have provided a compilation of transitions of importance to the search for $\alpha$ variation. They are E1 transitions from the ground state in several different atoms and ions, with wavelengths ranging from around $900-6000 \AA$, and require an accuracy of better than $10^{-4} \AA$. We also discuss isotope shift measurements that are needed in order to resolve systematic effects in the study.

Researchers who are interested in performing these measurements should contact the authors directly.

\section{References}

Dzuba, V. A., Flambaum, V. V., \& Webb, J. K. 1999, Phys. Rev. Lett., 82, 888

Flambaum, V. V. \& Berengut, J. C. 2009, Int. J. Mod. Phys. A, 24, 3342

Murphy, M. T., Flambaum, V. V., Webb, J. K., et al. 2004, Lect. Notes Phys., 648, 131

Murphy, M. T., Webb, J. K., \& Flambaum, V. V. 2007, Phys. Rev. Lett., 99, 239001

Murphy, M. T., Webb, J. K., \& Flambaum, V. V. 2008, Mon. Not. R. Astron. Soc., 384, 1053

Srianand, R., Chand, H., Petitjean, P., \& Aracil, B. 2004, Phys. Rev. Lett., 92, 121302 